\title{
ASSOCIAÇÃO ENTRE FLEBITE E RETIRADA DE CATETERES INTRAVENOSOS PERIFÉRICOS
}

\author{
Nelissa de Paula Magerote ${ }^{1}$, Maria Helena de Melo Lima², Juliana Bastoni Silva3 , Marisa Dibbern Lopes \\ Correia ${ }^{4}$, Silvia Regina Secoli ${ }^{5}$
}

${ }^{1}$ Graduanda do $8^{\circ}$ semestre do curso de Enfermagem da Faculdade de Ciências Médicas (FCM) da Universidade Estadual de Campinas (UNICAMP). E-mail: ne_depaula@yahoo.com.br

${ }^{2}$ Doutora em Biologia Funcional e Molecular. Professora Doutora do Curso de Enfermagem da FCM/UNICAMP. E-mail: mhmelolima@gmail.com

${ }^{3}$ Doutoranda do Programa de Pós-Graduação em Enfermagem na Saúde do Adulto da Escola de Enfermagem da Universidade de São Paulo (EEUSP). Enfermeira do Curso de Enfermagem da FCM/UNICAMP. E-mail: julianab@fcm.unicamp.br

${ }^{4}$ Mestre em Enfermagem. Professora Assistente I do Curso de Graduação em Enfermagem do Departamento de Medicina e Enfermagem Universidade Federal de Viçosa. E-mail: marisa.lopes@ufv.br

${ }^{5}$ Pós-Doutora em Farmacoepidemiologia. Professora Associado do Departamento de Enfermagem Médico-cirúrgica da EEUSP. E-mail: secolisi@usp.br

RESUMO: Estudo de coorte prospectivo, com objetivo de verificar a incidência de flebite em pacientes com cateter intravenoso periférico e identificar possíveis associações dessa complicação com variáveis relativas a estes cateteres. Foram avaliados 76 pacientes adultos internados em enfermaria de um hospital universitário, utilizando-se de uma ficha contendo variáveis relativas aos pacientes, ao cateter intravenoso periférico e à terapia farmacológica. A flebite foi avaliada segundo classificação proposta pela Infusion Nurses Society. Utilizou-se estatística descritiva e inferencial. Observou-se incidência de $25,8 \%$ de flebite, das quais $40 \%$ apresentaram manifestações clínicas de dor, com eritema e ou edema. Dentre os pacientes com flebite, $60 \%$ desenvolveram um episódio da complicação, e o tempo de permanência médio dos cateteres intravenosos periféricos com flebite foi de 3,10 dias. Constatou-se associação da flebite à retirada do cateter intravenoso periférico por ocorrência adversa $(p<0,001)$. São necessárias ações educativas e capacitação da equipe de enfermagem para o manejo da terapia intravenosa, principalmente no que tange à deteç̧ão precoce da flebite.

DESCRITORES: Infusões intravenosas. Flebite. Enfermagem. Cuidados de enfermagem. Cateterismo periférico.

\section{RELATION BETWEEN PHLEBITIS AND PERIPHERAL INTRAVENOUS CATHETER REMOVAL}

\begin{abstract}
The objective of this prospective study is to verify the incidence of phlebitis among patients with peripheral intravenous catheters and to identify possible relations between this complication and catheter-related variables. Seventy-six adult patients hospitalized in a Brazilian university hospital were evaluated, using charts with patient-related variables, peripheral intravenous catheters, and pharmacological therapy. Phlebitis was evaluated based on Infusion Nurses Society proposed classification. Inferential and descriptive statistics have been used. We observed $25.8 \%$ incidence of phlebitis, from which $40 \%$ presented pain occurrences, with erythema and edema. Among patients with phlebitis, $60 \%$ developed a complication episode, and the average period of permanence of peripheral intravenous catheters with phlebitis was 3.10 days. We observed phlebitis associations to adverse occurrences during the removal of peripheral intravenous catheters $(\mathrm{p}<0.001)$. Educational actions and professional training for nursing personnel are necessary for dealing with intravenous therapy, mainly on what is related to early detection of phlebitis.
\end{abstract}

DESCRIPTORS: Intravenous infusions. Phlebitis. Nursing. Nursing care. Peripheral catheterization.

\section{RELACIÓN ENTRE LA FLEBITIS Y LA RETIRADA DE CATÉTERES INTRAVENOSOS PERIFÉRICOS}

RESUMEN: Se trata de un estudio prospectivo para determinar la incidencia de flebitis en pacientes con catéter intravenoso periférico e identificar posibles asociaciones de esta complicación con las variables relacionadas con el catéter intravenoso periférico. Se evaluaron setenta y seis pacientes adultos hospitalizados en el sector de enfermería de un hospital universitario, mediante un formulario que contiene las variables relacionadas con los pacientes, el catéter intravenoso periférico y la terapia farmacológica. La flebitis fue evaluada de acuerdo a la clasificación propuesta por la Infusion Nurses Society. Se utilizó la estadística descriptiva e inferencial. La incidencia de flebitis fue del 25,8\%, de los cuales, el $40 \%$ presentó síntomas clínicos de dolor, con eritema y/o edema. Entre los pacientes con flebitis, el $60 \%$ desarrolló un episodio de complicación, y el tiempo medio de permanencia del catéter intravenoso periférico con flebitis fue 3,10 días. Se observó una relación de la flebitis con la retirada de catéter intravenoso periférico por eventos adversos ( $<<0,001)$. Se necesitan acciones educativas y capacitación del personal de enfermería para el manejo de la terapia intravenosa, especialmente en lo que respecta a la detección temprana de flebitis.

DESCRIPTORES: Infusiones intravenosas. Flebitis. Enfermería. Atención de enfermería. Cateterismo periférico. 


\section{INTRODUÇÃO}

No contexto atual, os Cateteres Intravenosos Periféricos (CIPs) representam um recurso indispensável na clínica, sendo utilizados predominantemente para infundir grandes volumes de líquidos, medicamentos e hemoderivados, bem como administrar agentes que são alterados pelo suco gástrico ou mal absorvidos pelo trato gastrintestinal. ${ }^{1}$ Desse modo, a inserção de CIP é um dos procedimentos mais frequentes da prática dos profissionais de enfermagem, sendo estes os principais responsáveis pelos cuidados relativos aos dispositivos e pela Terapia Intravenosa (TIV). Estima-se que mais de $60 \%$ dos pacientes admitidos para atendimento hospitalar requer TIV. ${ }^{2}$

Todavia, o uso dos CIPs encontra-se, muitas vezes, associado a complicações, as quais podem gerar maior carga de trabalho aos profissionais, ônus financeiro ao indivíduo, familiares, sistema de saúde e, principalmente, ser causa adicional de dor e sofrimento para o paciente. Essas complicações podem ser resultado direto da técnica de inserção do CIP ou relacionadas às propriedades físico químicas dos fluidos administrados. Entretanto, independentemente do fator gerador, as complicações locais são expressas por meio de hematoma, infiltração, extravasamento, obstrução do cateter e flebite. ${ }^{3-5}$

No âmbito hospitalar, a flebite é uma das complicações mais frequentes e considerada uma das principais falhas da infusão, que implicam na interrupção da TIV, sendo uma das causas preveníveis de morbimortalidade de pacientes. ${ }^{5}$

Flebite é o processo inflamatório da camada íntima das veias causado por irritação mecânica, química ou infecções bacterianas, cujas manifestações incluem dor, edema, hiperemia local e calor. Na evolução, pode, também, surgir cordão fibroso palpável, aumento da temperatura basal e, em casos infecciosos, presença de secreção purulenta no sítio de inserção do cateter. . $^{1,3}$

Pesquisas conduzidas nas décadas de 1980 e 1990 apontaram que a incidência dessa complicação foi bastante distinta. Estudo multicêntrico desenvolvido com 5.161 CIPs encontrou 2,5\% de flebite. ${ }^{6}$ Ensaio clínico conduzido com 1.054 CIPs indicou incidência de $30 \%$ e $45 \%$, com dois e três dias de cateterização, respectivamente. ${ }^{3}$ Revisão crítica acerca da epidemiologia da tromboflebite concluiu que a incidência variou de 25 a 35\%.7 Investigações mais recentes oriundas de hospitais universitários da Espanha e Turquia mostraram que a incidência foi de $2,7 \%$ e $54,5 \%$, respectivamente. ${ }^{4,8}$ No cenário brasileiro, os estudos conduzidos com pacientes hospitalizados em unidades clínicas e cirúrgicas mostraram incidência de 3\% a $20,6 \%{ }^{9-12}$

Os estudos relativos aos fatores associados ao desenvolvimento da flebite são, ainda, inconclusivos, porém apontam que o risco aumenta dependendo do tipo de material e calibre do $\mathrm{CIP}, 3,13$ do tempo de permanência do CIP, , 3,7,10,13-15 do local da punção, ${ }^{16}$ do uso de fármacos hipertônicos e pH ácido. ${ }^{8,17}$

Considerando o panorama, que a flebite representa um importante indicador da qualidade assistencial de enfermagem, e que no contexto nacional os estudos sobre o tema são, ainda, incipientes, os objetivos do presente estudo foram: a) Verificar a incidência de flebite em pacientes com CIP; e b) Identificar as possíveis associações dessa complicação com as variáveis relativas aos CIPs e à terapia farmacológica em pacientes internados em uma Enfermaria Geral de Adultos (EGA).

\section{MÉTODO}

Trata-se de um estudo de coorte prospectivo, realizado na EGA do Hospital de Clínicas (HC) da Universidade Estadual de Campinas (UNICAMP), no período de fevereiro a junho de 2009. O estudo foi aprovado pelo Comitê de Ética em Pesquisa da Instituição (Parecer no 1091/2008). Os pacientes elegíveis e que aceitaram participar do estudo foram orientados sobre os objetivos do estudo, o caráter voluntário da participação, sigilo, anonimato e assinaram o Termo de Consentimento Livre e Esclarecido.

A EGA possui 36 leitos e atende indivíduos adultos com afecções clínicas. O quadro de equipe de enfermagem é composto por 13 enfermeiros, cinco auxiliares e 31 técnicos de enfermagem. Há três turnos de trabalho e todos os profissionais de enfermagem realizam a cateterização do acesso venoso periférico.

A amostra foi, inicialmente, composta por 89 pacientes, os quais atenderam aos critérios de elegibilidade, quais sejam: idade igual ou superior a 18 anos, com nível de orientação preservado, segundo avaliação clínica (orientação temporal e espacial), estar apto para responder as questões verbais e ter sido puncionado com CIP na EGA. Todavia, houve perda de seguimento de 13 destes, sendo a amostra analisada constituída por 76 pacientes. 
O procedimento de inserção do CIP, adotado na Instituição, consiste na antissepsia da pele com algodão e álcool 70\% e na fixação (estabilização e curativo) do CIP com fita hipoalergênica (micropo$\left.\mathrm{re}^{\circledR}\right)$. Não há protocolo ou rotina de troca da fixação do CIP. Todavia, a troca de CIP ocorre, quando possível, em intervalo de 72 a 96 horas, de acordo com a recomendação da Comissão de Controle de Infecção Hospitalar do HC-UNICAMP, a qual segue o Centers for Disease Control and Prevention (CDC). ${ }^{18}$ Essa recomendação é revista quando o paciente apresenta rede venosa de difícil acesso.

Na coleta de dados, utilizou-se um instrumento constituído por variáveis relativas aos pacientes e aos CIP inseridos, quais sejam: idade, sexo, diagnóstico médico, tipo de CIP, material do CIP, calibre do CIP, localização anatômica da inserção do CIP, tempo de permanência do CIP, número de CIP inseridos e motivos da retirada do CIP. Essa última foi categorizada em: final da terapia farmacológica (alta hospitalar, fim do regime terapêutico) ou ocorrências adversas (flebite, infiltração, obstrução, remoção acidental do CIP).

$\mathrm{Na}$ tentativa de estabelecer relação entre terapia farmacológica e ocorrência de flebite, as prescrições médicas dos sujeitos foram avaliadas durante o período de permanência do CIP. Assim, foram coletados, também, os seguintes dados: nome do medicamento, diluente e modo de infusão.

Os medicamentos foram classificados segundo a Anatômico Terapêutico Químico (ATC), cuja estrutura é dividida em cinco níveis, sendo o primeiro nível subdividido em 14 grupos anatômicos principais, codificados por letras. Os níveis dois e três correspondem aos subgrupos terapêuticos e farmacológicos. O nível quatro corresponde ao grupo terapêutico, farmacológico e químico, e o último, nível cinco, representa a substância química. ${ }^{19}$ Os anti-infecciosos foram identificados quanto ao potencial flebitogênico, ou seja, quanto à capacidade de causar flebite. Utilizou-se literatura específica de medicamentos intravenosos, na qual a flebite encontra-se descrita no item reação adversa local. ${ }^{20}$

Para a avaliação da flebite utilizou-se a classificação proposta pela Infusion Nurses Society (INS), segundo a qual essa complicação apresenta graus de evolução, quais sejam: Grau zero: sem sintomas; Grau 1: eritema com ou sem dor local; Grau 2: dor com eritema e ou edema; Grau 3: dor com eritema e ou edema, com endurecimento e cordão fibroso palpável; Grau 4: dor com eritema e ou edema, com endurecimento e cordão fibroso palpável maior que $2,5 \mathrm{~cm}$ de comprimento e drenagem purulenta. ${ }^{21}$

O período de acompanhamento dos sujeitos ocorreu desde a punção do CIP até a retirada do mesmo. As avaliações do sítio de inserção para detecção dos sinais indicativos de flebite ocorreram diariamente no período da manhã, por um único observador, com início após 24 horas da punção, até a retirada do dispositivo, inclusive nas situações em que o dispositivo não foi usado. $\mathrm{Na}$ vigência de ocorrência de flebite, o CIP era retirado e um novo acesso era puncionado.

Os dados foram organizados em planilha do Excel ${ }^{\circledR} 2003$ do Windows XP ${ }^{\circledR}$ e exportados para o software Statistical Package for the Social Science (SPSS), versão 16.0, para análise estatística. Utilizou-se o teste de Qui Quadrado para verificar a diferença entre os grupos nas proporções das variáveis relativas aos CIPs. Nos casos em que não foi adequado o Qui Quadrado, utilizou-se razão de verossimilhança. Para comparação das medidas do tempo de permanência dos CIPs utilizou-se o teste de Mann Whitney. Adotou-se nível de significância de $\mathrm{p}<0,05$.

\section{RESULTADOS}

Dentre os 76 pacientes incluídos no estudo, $52,6 \%$ eram do sexo masculino, com média de idade de $54,4( \pm 17,8)$ anos, variando de 18 a 85 anos, $42,1 \%$ com problemas cardiorrespiratórios e 13,2\% com doenças renais. Essa casuística foi submetida à inserção de 155 CIP do tipo cateter sobre agulha, todos confeccionados em poliuretano - Vialon ${ }^{\circledR}$.

A média do número de CIP por paciente foi de 2,03. Dos pacientes, 44,7\% utilizaram um CIP, $25 \%$ dois CIP, $17,1 \%$ três CIP. Observou-se, ainda, que $7,9 \%$ e $5,3 \%$ dos pacientes foram puncionados com quatro e cinco CIPs, respectivamente.

Dentre os 155 CIPs, 40 sítios de inserção $(25,8 \%)$ desenvolveram flebite. Na análise da incidência dessa complicação, observou-se que $65 \%$ das ocorrências foram em cateteres de maior calibre, $18 \mathrm{G}$ e $20 \mathrm{G}$, inseridos predominantemente no lado esquerdo (55\%), no antebraço $(57,5 \%)$, com tempo de permanência menor ou igual a $72 \mathrm{~h}$ $(67,5 \%)$ e que não receberam a infusão de antibióticos flebitogênicos (80\%). Em 85\% dos casos com flebite, o motivo de retirada dos CIP foi ocorrência adversa, evidenciando-se associação significativa entre essa variável e a presença da complicação analisada $(\mathrm{p}<0,001)$ (Tabela 1$)$. 
Tabela 1 - Distribuição dos cateteres com e sem flebite segundo variáveis relacionadas aos cateteres. Campinas-SP, 2009

\begin{tabular}{|c|c|c|c|c|}
\hline Variáveis relativas aos cateteres & $\begin{array}{c}\text { Cateteres com flebite } \\
\text { n (\%) }\end{array}$ & $\begin{array}{c}\text { Cateteres sem } \\
\text { flebite } \\
\mathrm{n}(\%)\end{array}$ & $\begin{array}{l}\text { Total } \\
\text { n (\%) }\end{array}$ & $\mathrm{p}$ valor \\
\hline Calibre* $^{*}$ & & & & $0,711^{\dagger}$ \\
\hline $18 \mathrm{G}$ e $20 \mathrm{G}$ & $26(65,0)$ & $69(60,0)$ & $95(61,3)$ & \\
\hline 22 G e 24 G & $14(35,0)$ & $46(40,0)$ & $60(38,7)$ & \\
\hline Lado da inserção & & & & $0,901^{\dagger}$ \\
\hline Direito & $18(45,0)$ & $55(47,8)$ & $73(47,1)$ & \\
\hline Esquerdo & $22(55,0)$ & $60(52,2)$ & $82(52,9)$ & \\
\hline Região anatômica da inserção & & & & $0,056^{\ddagger}$ \\
\hline Antebraço & $23(57,5)$ & $45(39,1)$ & $68(43,9)$ & \\
\hline Fossa antecubital & $3(7,5)$ & $8(7,0)$ & $11(7,1)$ & \\
\hline Punho & $6(15,0)$ & $30(26,1)$ & $36(23,2)$ & \\
\hline Mão & $5(12,5)$ & $30(26,1)$ & $35(22,6)$ & \\
\hline Braço & $3(7,5)$ & $2(1,7)$ & $5(3,2)$ & \\
\hline Tempo de permanência & & & & $0,400^{\dagger}$ \\
\hline$<$ ou igual $72 \mathrm{~h}$ & $27(67,5)$ & $67(58,3)$ & $94(60,6)$ & \\
\hline$>72 \mathrm{~h}$ & $13(32,5)$ & $48(41,7)$ & $61(39,4)$ & \\
\hline Motivo da retirada & & & & $<0,001^{\dagger}$ \\
\hline Ocorrência adversa & $33(82,5)$ & $36(31,3)$ & $69(44,5)$ & \\
\hline Data de troca do CIP & $4(10,0)$ & $12(10,4)$ & $16(10,3)$ & \\
\hline Final da terapia & $3(7,5)$ & $67(58,3)$ & $70(45,2)$ & \\
\hline Uso de anti-infeccioso flebitogênico & & & & $0,601^{\dagger}$ \\
\hline Não & $32(80,0)$ & $98(85,2)$ & $130(83,9)$ & \\
\hline Sim & $8(20,0)$ & $17(14,8)$ & $25(16,1)$ & \\
\hline
\end{tabular}

* = Gauge; $†$ = Qui-Quadrado; $\ddagger$ = Razão de verossimilhança.

O tempo de permanência médio dos CIPs foi de 3,15 dias; para aqueles que desenvolveram flebite foi 3,10 dias, variando de um a seis dias (Tabela 2).

Tabela 2 - Análise descritiva do tempo de permanência dos cateteres venosos periféricos segundo ocorrência ou não de flebite. CampinasSP, 2009

\begin{tabular}{lccccc}
\hline Flebite & $\mathbf{n}$ & Mediana & $\begin{array}{c}\text { Média } \\
\mathbf{( \pm \text { desvio }} \text { padrão) }\end{array}$ & Mínimo Máximo \\
\hline Não & 115 & 3,00 & $3,17( \pm 1,34)$ & 1 & 6 \\
Sim & 40 & 3,00 & $3,10( \pm 1,33)$ & 1 & 6 \\
\hline Total & 155 & 3,00 & $3,15( \pm 1,33)$ & 1 & 6 \\
\hline
\end{tabular}

Dentre os 40 sítios de inserção que desenvolveram flebite, $40 \%$ apresentaram manifestações clínicas de dor, com eritema e ou edema (Tabela 3).

Tabela 3 - Distribuição das flebites segundo grau de evolução. Campinas-SP, 2009

\begin{tabular}{lr}
\hline Graus de flebite & $\mathbf{n ~ ( \% )}$ \\
\hline Grau 1 & $13(32,5)$ \\
Grau 2 & $16(40,0)$ \\
Grau 3 & $1(2,5)$ \\
Grau 4 & $10(25,0)$ \\
\hline
\end{tabular}

As 40 ocorrências de flebites foram distribuídas do seguinte modo: 24 (60\%) pacientes desen- 
volveram um episódio de flebite, três tiveram três episódios (22,5\%), um apresentou dois (5\%) e um paciente cinco flebites $(12,5 \%)$.

Durante o período de estudo os 155 CIPs foram utilizados para administração de 28 medicamentos distintos, pertencentes a seis classes terapêuticas, quais sejam: J, Anti-infecciosos (16; 57,2\%); A, Trato alimentar e metabolismo (5; 17,8\%); C, Cardiovascular (3; 10,7\%); N, Sistema nervoso $(2 ; 7,1 \%) ; \mathrm{H}$, Sistema hormonal $(1 ; 3,6 \%)$; e R, Sistema respiratório $(1 ; 3,6 \%)$. Entre os anti-infecciosos destacaram-se aqueles com potencial flebitogênico $(11 ; 68,7 \%)$ e o grupo dos beta-lactâmicos (10; 62,5\%). Todos os anti-infecciosos foram diluídos, predominantemente, em solução fisiológica (SF 0,9\%), cujo volume variou de 100 a $500 \mathrm{ml}$, e o período de infusão variou de $30 \mathrm{minu}-$ tos a uma hora.

\section{DISCUSSÃO}

No presente estudo, observou-se que 25,8\% dos sítios de inserção de CIP desenvolveram flebite. Essa incidência, apesar de ser maior que o padrão aceito de $5 \%$ pela INS americana, ${ }^{21}$ encontra-se próxima àquelas identificadas $(20,6 \%$ a $27 \%$ ) em estudos prévios realizados com pacientes adultos. ${ }^{10,22}$ A literatura aponta taxas bastante distintas que variam de $2,7 \%$ a $54,5 \%{ }^{4,7-12,23}$ Essa discrepância pode estar relacionada à dificuldade de padronizar o diagnóstico e às nuances relativas aos graus de flebite, que envolvem sinais como eritema, edema, endurecimento, cordão fibroso, que podem apresentar leituras diferentes aos olhos dos profissionais. Adicionalmente, há que se considerar a influência das características da amostra, bem como da equipe de trabalho, na ocorrência dessa complicação.

A avaliação do grau de flebite apontou que $72 \%$ dos sítios não apresentaram endurecimento, cordão fibroso palpável e drenagem purulenta, que são indicadores de graus mais avançados da complicação. Esses achados estão em consonância com outros estudos que utilizaram, também, a escala da INS. ${ }^{4,12}$ Entretanto, o fato de $25 \%$ dos acessos terem evoluído para o Grau 4, mostra que é fundamental a implementação de estratégias educativas que permitam a prevenção ou identificação precoce (Grau 1) dessa complicação por parte da equipe de enfermagem. Neste estudo, a complicação pode ter evoluído a graus mais graves, porque os CIP foram fixados com fita hipoalergênica, dificultando a avaliação diária do sítio de inserção.
O tempo de permanência e o calibre do CIP, preditores da flebite apontados por inúmeros autores, ${ }^{3,7,10,13-15}$ não apresentaram impacto no presente estudo. Esse achado discordante pode ser devido a dois motivos: todos os CIPs usados eram de poliuretano - Vialon ${ }^{\circledR}$, e o tempo médio de permanência foi de 3,15 dias, ou seja, 72 h. O Vialon ${ }^{\circledR}$ é um polímero elastomérico que possui baixa incidência trombogênica e é mais flexível, especialmente quando atinge a temperatura corporal, por isso permite maior tempo de canulação do acesso, apresentando menor risco de flebite. ${ }^{1,3,7,13}$ Pesquisas mostraram que a taxa dessa complicação local não aumenta com o tempo de permanência do CIP. ${ }^{23-25}$

Quanto à região anatômica e a ocorrência de flebite, não se observou associação significativa $(p=0,059)$. O antebraço foi o local de punção mais utilizado pela equipe de enfermagem. A preferência por essa região, também evidenciada em outras investigações, encontra-se relacionada à presença de veias calibrosas e longas, como cefálica e basílica, que possibilitam a inserção de CIP mais calibrosos. ${ }^{4,12,24} \mathrm{O}$ fato de esses acessos terem sido puncionados principalmente com CIP de 18G e 20G, pode ter, de certo modo, evitado a flebite. Atualmente não existe consenso acerca da região anatômica ideal ou de menor risco de flebite. A inserção de $\mathrm{CIP}$ de maior calibre em veias tortuosas e de menor lúmen como mãos e punhos pode causar irritação mecânica na parede vascular, levando à flebite. ${ }^{1,16,25}$ Todavia, punções na fossa antecubital e na região do antebraço, por serem as mais comumente utilizadas, apresentam maiores taxas de flebite em relação às do dorso da mão e punho. ${ }^{1,3-4}$

A despeito dos anti-infecciosos representarem a classe terapêutica mais utilizada $(57,2 \%)$, e destes $68,7 \%$ apresentarem potencial flebitogênico, sendo o grupo dos beta-lactâmicos $(62,5 \%)$ o mais utilizado, não houve associação significativa entre essa variável e flebite. Esses achados corroboram estudos prévios que também não verificaram essa relação. ${ }^{4,8,23} \mathrm{O}$ risco relacionado aos anti-infecciosos, ainda que beta-lactâmicos, sabidamente capazes de ocasionar irritação do endotélio vascular, pode ter sido amenizado pela diminuição da osmolaridade da solução. A redução da concentração do soluto decorrente do grande volume de diluente usado (100 a $500 \mathrm{ml}$ ), associada à baixa frequência $(20 \%)$ de uso de anti-infecciosos flebitogênicos, pode ter contribuído para a não instalação da flebite.

Observou-se associação significativa entre motivo de retirada do CIP e flebite $(p<0,001)$. A 
maioria dos CIPs com flebite $(82,5 \%)$ foi retirada devido a ocorrências adversas, dentre as quais a própria flebite. Esse dado permite evidenciar que complicações locais como flebite, infiltração e obstrução, apesar de preveníveis, são as principais responsáveis pela interrupção da TIV. Essas ocorrências encontram-se intimamente relacionadas ao padrão de cuidado realizado pela equipe de enfermagem. Pesquisa verificou que os cuidados recebidos pelo paciente durante a TIV foram insatisfatórios, ou seja, havia problemas relacionados à instabilidade do cateter, perda do acesso venoso, presença de sangue na parte externa do dispositivo ou no curativo. ${ }^{26}$

Adicionalmente, essas ocorrências devem ser analisadas num cenário mais amplo, cujo impacto pode ser clínico, acarretando sofrimento desnecessário para o paciente e comprometendo a segurança, e também o econômico, tendo em vista que, na vigência de seguimento da terapia, novas punções fazem-se necessárias. Além disso, o aumento da gravidade de complicações como flebite podem demandar a adoção de terapias específicas, elevando o tempo de hospitalização do paciente. Nesse contexto, enfermeiras com conhecimentos sólidos em TIV podem fazer a diferença na assistência. Estudos mostraram que a taxa de complicações locais dos pacientes foi menor quando foram cuidados pelo grupo de enfermeiras treinadas em TIV. .77,28 Desse modo, a elaboração de protocolos pautada em evidências poderá reduzir a variabilidade da prática dos profissionais de enfermagem, proporcionar melhoria da qualidade e reduzir riscos e complicações da TIV.

Esse estudo apresentou limitações. Houve perdas de seguimento de alguns pacientes, inviabilizando a identificação das flebites pós infusionais. A fixação do CIP com fita hipoalergênica não estéril, que representa a realidade de grande parte dos hospitais públicos brasileiros, dificultou a avaliação diária do sítio de inserção. Não foi possível demonstrar a relação entre anti-infecciosos flebitogênicos e flebite, uma vez que o volume de diluição utilizado em muitos agentes foi superior ao recomendado nos dicionários de especialidades farmacêuticas. Ademais o estudo não controlou a variável frequência de uso do CIP para infusão de medicamentos, que supostamente pode elevar o risco de flebite.

\section{CONCLUSÃO}

A incidência de flebite em adultos internados em clínica médica foi cerca de cinco vezes maior $(25,8 \%)$ que a aceita pela INS, e o motivo de reti- rada por ocorrências adversas foi a única variável que apresentou associação com a ocorrência da flebite. Esses achados apontam que são necessárias ações educativas e capacitação da equipe de enfermagem no que concerne às especificidades da TIV, principalmente no que tange a detecção precoce da flebite.

\section{REFERENNCIAS}

1. Santos Netto P, Secoli SR. A flebite enquanto complicação da terapia intravenosa: estudo de revisão. Rev Paul Enf. 2005 Jul-Dez; 23(3/4):254-9.

2. Bregenzer T, Conen D, Sakmann P, Windmer AF. Is routine replacement of peripheral intravenous catheters necessary? Arch Intern Med. 1998 Jan; 158:151-6

3. Maki DG, Ringer M. Risk factors for infusion related phlebitis with small peripheral venous catheter. Ann Intern Med. 1991May; 114(10):845-54.

4. Uslusay E, Mete S. Predisposing factors to phlebitis in patients with peripheral intravenous catheters: a descriptive study. J Am Acad Nurse Pract. 2008 Apr; 20(4):172-80.

5. Gabriel J. Infusion therapy part one: minimising the risks. Nurs Stand. 2008 Apr; 22(31):51-6.

6. Tager IB, Ginsberg MB, Ellis SE, Walsh NE, Dupont I, Simchen E, et al. An epidemiologic study of the risks associated with peripheral intravenous catheters. Am J Epidemiol. 1983 Dec; 118(6):839-51.

7. Tagalakis V, Kahn SR, Liberman M, Blostein M. The epidemiology of peripheral vein infusion thrombophlebitis: a critical review. Am J Med. 2002 Aug; 113(2):146-51.

8. Ferrete-Morales C, Vázquez-Pérez MA, SanchezBerna M, Gilabert-Cerro I, Corzo-Delgado JE, Pineda-Vergara JA, et al. Incidência de flebitis secundaria por cateter venoso de acceso periférico e impacto de un protocolo de manejo. Enferm Clin. 2010 Jan-Feb; 20(1):3-9.

9. Nascimento EMF, Souza MF, Latorre MRDO, Aguilar-Nascimento JE. Efeito de padrões para a prática da terapia intravenosa incluindo inovações tecnológicas na sobrevida de inserção intravenosas periféricas. Acta Paul Enferm. 2000 Set-Dez; 13(3):66-75.

10. Nascimento EMF, Souza MF. Infiltração em terapia intravenosa através de veia periférica. Acta Paul Enferm. 1996 Jan-Apr; 9(1):53-60.

11. Pereira RCC, Zanetti ML, Ribeiro KP. Motivos de interrupção da terapia endovenosa relacionada ao tempo de permanência do dispositivo venoso periférico in situ. Rev Gaúcha Enferm. 2002 Jan; 23(1):70-83.

12. Ferreira LR, Pedreira MLG, Diccini S. Flebite no pré e pós-operatório de pacientes neurocirúrgicos. Acta Paul Enferm. 2007 Jan-Mar; 20(1):30-6. 
13. Karadag A, Görgülü S. Effect of two different short peripheral catheter materials on phlebitis development. J Intraven Nurs. 2000 MayJun;23(3):158-66.

14. Idvall E, Gunningberg L. Evidence for elective replacement of peripheral intravenous catheter to prevent thrombophlebitis: a systematic review. J Adv Nurs. 2006 Sep; 55(6):715-22.

15. Carballo M, Llinas M, Feijoo M. Flebitis em cateteres periféricos (I): incidência y factores de riesgo. Rev Enferm. 2004 Sep; 27(9):25-32.

16. Karadeniz G, Kutlu N, Tatlisumak E, Özbakkaloglu B. Nurse's knowledge regarding patients with intravenous catheters and phlebitis interventions. J Vasc Nurs. 2003 Jun; 21(2):44-7.

17. Lanbeck P, Odenholt I, Riesbeck K. Dicloxacillin and erythromycin at high concentrations increase ICAM-1 expression by endothelial cells: a possible factor in the pathogenesis of infusion phlebitis. J Antimicrob Chemother. 2004 Feb; 53(2):174-9.

18. Centers for Disease Control and Prevention. Guidelines for the prevention of intravascular catheter-related infections. MMWR. 2002 Aug; 51:1-32.

19. WHO-World Health Organization. Anatomical therapeutical chemical (ATC) classification index with defined daily doses (DDDs) [Internet]. Geneva; c2007. [atualizado 2006 Dez 16; acesso 2007 Mar 16]. Disponível em: http://www.whocc.no/ atc_ddd_index/

20. Gahart BL, Nazareno AR. Intravenous medications. 26a ed. St. Louis (US): Elsevier Mosby; 2010.
21. Infusion Nursing Standards of Practice. J Infus Nurs 2006 Jan-Feb; 29:S1-92.

22. Grüne F, Schrappe M, Basten J, Wenchel HM, Tual E, Stützer H. Phlebitis rate and time kinetics of short peripheral intravenous catheters. Infection. 2004 Feb; 32(1):30-2.

23. Cornely OA, Bethe U, Pauls R, Waldschmidt D. Peripheral teflon catheters: factors determining incidence of phlebitis and duration of cannulation. Infect Control Hosp Epidemiol. 2002 May; 23(5):249-53.

24. Dillon MF, Curran J, Martos R, Walsh C, Walsh J, Al-Azawi D, et al. Factors that affect longevity of intravenous cannulas: a prospective study. QJM. 2008 Sep; 101(9):731-5.

25. Mazzola J, Schott-Baer D, Addy L. Clinical factors associated with the development of phlebitis after insertion of a peripherally inserted central catheter. J Infuven Nurs. 1999 Jan-Feb; 22(1):36-42. Erratum in: J Intraven Nurs 1999; 22(2):60.

26. Pereira RCC, Zanetti, ML. Complicações decorrentes da terapia intravenosa em pacientes cirúrgicos. Rev Latino-am enfermagem. 2000 Out; 8(5):21-7.

27. Soifer NE, Borzak S, Edlin BR, Weinstein RA. Prevention of peripheral venous catheter complications with an intravenous therapy team: a randomised controlled trial. Arch Intern Med. 1998 Mar; 158(5):473-7.

28. Karadag A, Görgülü S. Devising an intravenous fluid therapy protocol and compliance of nurses with the protocol. J Intraven Nurs. 2000 Jul-Aug; 23(4):232-8. 